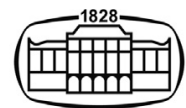

AKADÉMIAI KIADÓ

Journal of Behavioral Addictions

9 (2020) 3, 642-653

D01:

$10.1556 / 2006.2020 .00065$

(c) 2020 The Author(s)

\section{FULL-LENGTH REPORT}

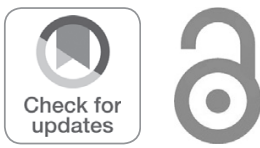

\title{
More stringent criteria are needed for diagnosing internet gaming disorder: Evidence from regional brain features and whole-brain functional connectivity multivariate pattern analyses
}

\author{
GUANG-HENG DONG ${ }^{1,2,3^{*}}$ (1), ZILIANG WANG ${ }^{4}$, \\ HAOHAO DONG ${ }^{5}$, MIN WANG $^{1,2,3}$, YANBIN ZHENG ${ }^{1,2,3}$, \\ SHUER YE ${ }^{1,2,3}$, JIALIN ZHANG ${ }^{5}$ and \\ MARC N. POTENZA ${ }^{6,7,8,9^{*}}$
}

\footnotetext{
${ }^{1}$ Center for Cognition and Brain Disorders, The Affiliated Hospital of Hangzhou Normal University, Hangzhou, PR China

${ }^{2}$ Zhejiang Key Laboratory for Research in Assessment of Cognitive Impairments, Hangzhou, Zhejiang Province, PR China

${ }^{3}$ Institute of Psychological Science, Hangzhou Normal University, Hangzhou, PR China

${ }^{4}$ School of Psychology, Beijing Normal University, Beijing, PR China

${ }^{5}$ Department of Psychology, Zhejiang Normal University, Jinhua, PR China

${ }^{6}$ Department of Psychiatry, Child Study Center, Yale University School of Medicine, New Haven, CT, USA

${ }^{7}$ Department of Neuroscience, Yale University School of Medicine, New Haven, CT, USA

${ }^{8}$ Connecticut Council on Problem Gambling, Wethersfield, CT, USA

${ }^{9}$ Connecticut Mental Health Center, New Haven, CT, USA
}

Received: March 6, 2020 • Revised manuscript received: April 10, 2020; August 15, 2020 - Accepted: September 2, 2020 Published online: October 7, 2020

\begin{abstract}
Background: Internet gaming disorder (IGD) is included in the DSM-5 as a provisional diagnosis. Whether IGD should be regarded as a disorder and, if so, how it should be defined and thresholded have generated considerable debate. Methods: In the current study, machine learning was used, based on regional and interregional brain features. Resting-state data from 374 subjects (including 148 IGD subjects with DSM-5 scores $\geq 5$ and 93 IGD subjects with DSM-5 scores $\geq 6$ ) were collected, and multivariate pattern analysis (MVPA) was employed to classify IGD from recreational game use (RGU) subjects based on regional brain features ( $\mathrm{ReHo}$ ) and communication between brain regions (functional connectivity; FC). Permutation tests were used to assess classifier performance. Results: The results demonstrated that when using DSM- 5 scores $\geq 5$ as the inclusion criteria for IGD subjects, MVPA could not differentiate IGD subjects from RGU, whether based on ReHo or FC features or by using different templates. MVPA could differentiate IGD subjects from RGU better than expected by chance when using DSM-5 scores $\geq 6$ with both ReHo and FC features. The brain regions involved in the default mode network and executive control network and the cerebellum exhibited high discriminative power during classification. Discussion: The current findings challenge the current IGD diagnostic criteria thresholding proposed in the DSM-5, suggesting that more stringent criteria may be needed for diagnosing IGD. The findings suggest that brain regions involved in the default mode network and executive control network relate importantly to the core criteria for IGD.
\end{abstract}

${ }^{*}$ Corresponding authors.

E-mail: dongguangheng@hznu.edu.cn, marc.potenza@yale.edu

\section{KEYWORDS}

internet gaming disorder, diagnosing criteria, MVPA, ReHo, functional connectivity 


\section{INTRODUCTION}

Because internet gaming disorder (IGD) has been associated with severe negative consequences, such as impaired psychological states, social deficits, and poor academic performance (Ioannidis et al., 2019; Przybylski, Weinstein, \& Murayama, 2017; Yao et al., 2017; Zheng et al., 2019), the American Psychiatric Association has included IGD as a potential diagnosable psychiatric disorder (DSM-5, https:// www.psychiatry.org/psychiatrists/practice/dsm). More recently, the eleventh edition of The International Classification of Diseases (ICD-11) has included criteria for gaming disorder (ICD-11, https://icd.who.int/browse11/1-m/en). The DSM-5 proposed nine criteria for diagnosing IGD that were adapted from gambling disorder and that have been widely used in studies and clinical contexts (Petry \& O'Brien, 2013; Petry et al., 2014), although evidence supporting their validity has been debated.

However, the direct transposition of IGD criteria from established diagnostic criteria for gambling disorder and the inclusion of dimensions such as tolerance or withdrawal have generated debate and controversy (Deleuze et al., 2017; Griffiths et al., 2016; Kardefelt-Winther et al., 2017; Petry et al., 2016). Evidence regarding features of IGD varies across studies (Brand et al., 2019; Kiraly \& Demetrovics, 2017). For example, prevalence estimates of IGD vary widely and range from less than $1 \%$ to approximately $50 \%$ (Petry \& O'Brien, 2013), with somewhat more narrow ranges (0.727.7\%) reported internationally (Mihara \& Higuchi, 2017; Rehbein, Kliem, Baier, Mossle, \& Petry, 2015) and from 0.3 to $1 \%$ in an online survey (Przybylski et al., 2017). Some differences relate to methodological approaches and varying definitions of IGD. In addition to prevalence, potential predictive factors (Dong, M. Wang, et al., in press; Dong, Zheng, et al., 2018; Strittmatter et al., 2016; Wartberg, Kriston, Zieglmeier, Lincoln, \& Kammerl, 2019) and negative effects (Dong, Liu, Zheng, Du, \& Potenza, 2019; Dong, Wang, Du, \& Potenza, 2018; van den Eijnden, Koning, Doornwaard, van Gurp, \& Ter Bogt, 2018) of IGD have been debated, and whether IGD could potentially be regarded as a mental disorder remains controversial (Aarseth et al., 2017; Potenza, 2018; Quandt, 2017; van Rooij et al., 2018). These debates suggest that further research is needed into IGD (Van Rooij \& Kardefelt-Winther 2017), with investigations needed to investigate its defining features and how it may be differentiated from recreational game use (RGU). Understanding the pathophysiology of IGD may provide insight into these debates.

Studies of IGD have suggested specific neural underpinnings. For example, brain regions involved in reward processing (including the nucleus accumbens (NAcc) and striatum (Brand et al., 2019; Dong, M. Wang, H., Zheng et al., 2020; Ziliang Wang et al., 2018) and executive function (including the anterior cingulate cortex (ACC), orbitofrontal cortex (OFC), inferior frontal gyrus (IFGs) and dorsolateral prefrontal cortex (DLPFC) (Dong, Wang, Zhang, Du, \& Potenza, 2019; Zheng et al., 2019) have been implicated. Relatively decreased engagement of executive control circuits and increased responses to gaming cues in rewardrelated brain regions have been observed, suggesting possible aberrant neural processing and functional coordination among these brain regions (Dong, Huang, \& Du, 2011; Palaus, Marron, Viejo-Sobera, \& Redolar-Ripoll, 2017; M. Wang, Dong, Zheng, Du, \& Dong, 2020).

In addition to task-based studies, resting-state fMRI has been employed to investigate regional and large-scale neural network function and dysfunction. During rest, low-frequency $(0.01-0.08 \mathrm{~Hz}$ ) blood oxygen level-dependent (BOLD) fluctuations in functional magnetic resonance imaging (fMRI) signals may relate to spontaneous neuronal activity, and correlational analytic approaches have proven effective for measuring functional connectivity (FC) network alterations in neuropsychiatric conditions including addictions (Park et al., in press; M. Wang, N. Zeng et al., in press; Z. Wang et al., 2019). Resting-state FC measured by the correlation between two fMRI time series has been used for the discrimination of psychiatric disorders (Biswal et al., 2010; Cai, Griffiths, Korgaonkar, Williams, \& Menon, in press; Yan et al., 2019). In dealing with resting-state data, the regional homogeneity (ReHo) also a commonly used method of rs-fMRI data analysis, which reflects the synchronized neural activity in functionally related brain regions. It has also been considered an important index for changes in spontaneous neural activity in the resting brain (Tian, Ren, \& Zang, 2012; Zang, Jiang, Lu, He, \& Tian, 2004).

Resting-state fMRI could enhance the understanding of pathophysiological mechanisms underlying failures to control gaming impulses in IGD (Park et al., in press; Y. Wang, Wu, Luo, Zhang, \& Dong, 2017; Z. Wang et al., 2019). Studies have also suggested dysregulation of distributed neural networks encompassing executive control over cravings rather than the functional breakdown of a single discrete brain region (Dong, Wang, Wang, Du, \& Potenza, 2019; Park et al., in press; R. Wang et al., 2019). Abnormal (i.e., differing from healthy comparison subjects) executive control and reward processing have been reported in IGD using resting-state fMRI FC, which detects synchronized spontaneous activity among anatomically distinct regions or networks. For example, studies have reported decreased FC among executive-control and reward-related brain regions (Chen et al., 2016; Han et al., 2018; Kim et al., 2019; Yuan et al., 2017), suggesting imbalanced networks relating to executive control and reward processes. Theoretical models of IGD have proposed that executive control and reward circuits contribute importantly and that the trade-off between these circuits may promote poorly controlled behaviors in IGD and other addictive disorders (Brand et al., 2019; Dong \& Potenza, 2014).

Although these studies provide insight into biological mechanisms underlying IGD, there exist significant study limitations. First, regional analysis may only identify brain features related to specific discrete brain regions; this type of analysis may not provide a neural-network-level view of the brain. Second, seed-based FC is limited to obtaining information related to the selected regions of interest, making it difficult to understand whole-brain patterns of FC (van den 
Table 1. Demographics and clinical characteristics of all subjects

\begin{tabular}{|c|c|c|c|}
\hline & $\begin{array}{c}\text { IGD } \\
N=148 \\
\text { Male }=86 \\
\text { Female }=62\end{array}$ & $\begin{array}{c}\text { RGU } \\
N=226 \\
\text { Male }=141 \\
\text { Female }=85\end{array}$ & $P$-value \\
\hline \multicolumn{4}{|l|}{ Demographics } \\
\hline Age (Year) $(M \pm S D)$ & $21.25 \pm 2.455$ & $21.60 \pm 2.484$ & 0.185 \\
\hline Years of education $(M \pm S D)$ & $14.56 \pm 1.494$ & $14.61 \pm 1.408$ & 0.773 \\
\hline \multicolumn{4}{|l|}{ Clinical characteristics $(M \pm S D)$} \\
\hline IAT score & $64.91 \pm 8.618$ & $39.21 \pm 10.62$ & 0.000 \\
\hline DSM score & $5.969 \pm 1.045$ & $2.529 \pm 1.417$ & 0.000 \\
\hline Gaming time (Hours/Week) & $8.264 \pm 3.641$ & $6.146 \pm 3.063$ & 0.000 \\
\hline Gaming history (Year) & $3.705 \pm 0.6632$ & $3.745 \pm 0.664$ & 0.594 \\
\hline
\end{tabular}

Abbreviations: IGD, Internet Gaming Disorder; RGU, recreational game use; M, mean; SD, standard deviation; IAT, Internet Addiction Test; DSM, number of DSM-5 criteria.

Heuvel \& Hulshoff Pol, 2010). Third, traditional group-level statistical methods do not provide a mechanism for evaluating the discriminative power of identified connections at the individual level (Craddock, Holtzheimer, Hu, \& Mayberg, 2009).

Multivariate pattern analysis (MVPAs) may overcome some of these limitations and provide insight into IGD as a diagnostic entity. MVPA has recently been utilized to investigate potential brain signatures for clinical diagnoses in mental disorders (Woo, Chang, Lindquist, \& Wager, 2017). The theoretical hypothesis is that if an MVPA-based classifier can label new samples with better-than-random accuracy, then the two populations are likely to be different, and the classifier may capture population-related differences (Erickson, Korfiatis, Akkus, \& Kline, 2017; Zeng et al., 2012). In MVPA, the support vector machine (SVM) is a commonly used approach. MVPA has successfully distinguished people with major depression from non-affected individuals (Zeng et al., 2012) and identified putative neuroimaging makers for obsessive-compulsive disorder (Bruin, Denys, \& van Wingen, 2019) and biological markers for bulimia nervosa (Cyr, Yang, Horga, \& Marsh, 2018). MVPA has also successfully been used to differentiate people with substance addictions from those without based on ReHo (Y. Zhang et al., 2011). While MVPA has proven useful in many psychiatric disorders, it has yet to be applied to IGD (Z. Wang, Dong, Du, Zhang, \& Dong, 2020).

To date, it is unknown whether MVPA can capture regional or whole-brain resting-state FC patterns to discriminate and identify individuals with IGD from those with RGU at the individual subject level with a high degree of accuracy. MVPAs abilities may be valuable in specifying neural mechanisms underlying behavioral features of IGD and providing additional information to advance our understanding of IGDs pathophysiology and diagnostic characteristics.

We aimed to use regional brain features ( $\mathrm{ReHo}$ ) and the FC to determine whether MVPA could distinguish IGD subjects from RGU based on current diagnostic criteria for IGD. To investigate, we used MVPA to determine whether
IGD subjects may be differentiated from those with RGU; (2) whether the current proposed diagnostic criteria may link to MVPAs propensity to distinguish IGD and RGU individuals; and, (3) if so, which regions show high discriminative power in discriminating IGD and RGU subjects.

\section{METHODS AND MATERIALS}

\section{Participant selection}

Valid resting-state data from 374 subjects (148 IGD subjects (DSM-5 score $\geq 5$ ), with 93 IGD subjects scoring $\geq 6$ ), and 226 RGU subjects) scanned between April 2016 and April 2019 were selected for the current study. Exclusion criteria (e.g., incomplete information, poor spatial normalization, incomplete brain coverage, excessive head motion) and final inclusions are provided in Table 1.

Criteria for selection of IGD and RGU subjects have been reported in our previous studies (Dong, M. Wang et al., in press; Dong, M. Wang, Z. Wang et al., 2020) and are described briefly below. IGD diagnosis was determined based on scores of 50 or more on Young's internet addiction test (IAT, www.netaddiction.com) (Young, 2009) and, concurrently, by meeting the proposed DSM-5 IGD criteria (Petry et al., 2014) (Table 1). RGU participants were required to meet fewer than 5 of the 9 proposed DSM- 5 criteria for IGD and to score less than 50 on Young's IAT.

All participants were right-handed and were university students recruited through advertisements. All participants provided written informed consent and underwent structured psychiatric interviews (using the Mini-International Neuropsychiatric Interview (MINI)) (Lecrubier et al., 1997) performed by an experienced psychiatrist. All participants were free of psychiatric disorders (including major depression, anxiety disorders, schizophrenia, and substancedependence disorders) as assessed by the MINI. Prior to fMRI, participants were asked to complete a 10-item gaming urge questionnaire based on a tobacco craving questionnaire 
using a 10-point response scale (Cox, Tiffany, \& Christen, 2001).

\section{Data acquisition}

During fMRI, subjects were instructed to keep their eyes closed, relax, remain awake and perform no specific cognitive exercises. Resting-state functional data (T2*-weighted images) were acquired before any tasks were performed using a 3T Siemens Trio MRI scanner. Earplugs and a head coil with foam pads were used to minimize machine noise and head motion. Specific parameters are as follows: repetition time $=2,000 \mathrm{~ms}$, interleaved 33 axial slices, echo time $=30 \mathrm{~ms}$, thickness $=3.0 \mathrm{~mm}$, flip angle $=90^{\circ}$, field of view $($ FOV $)=220 \mathrm{~mm} \times 220 \mathrm{~mm}$, matrix $=64 \times 64$. To minimize head movement, all subjects' heads were fixed with foam padding. Each fMRI scan lasted $7 \mathrm{~min}$ and included 210 imaging volumes.

\section{Data preprocessing}

Resting-state data analysis was performed using DPABI (Yan, Wang, Zuo, \& Zang, 2016), a user-friendly pipeline package that incorporates recent research advances in headmotion control and measurement standardization. Preprocessing was performed using a standard approach that consisted of the following steps: (1) the initial $10 \mathrm{vol}$ were discarded, and slice-timing correction was performed; (2) the time series of images for each subject were realigned using a six-parameter (rigid-body) linear transformation; (3) individual T1-weighted images were co-registered to the mean functional image using a 6-degrees-of-freedom linear transformation without re-sampling and was then segmented into gray matter (GM), white matter (WM) and cerebrospinal fluid (CSF); (4) transformations from individual native space to the MNI space were computed with the DARTEL tool; (5) the Friston 24-parameter model was used to regress out head-motion effects with global signal regression (Fox, Zhang, Snyder, \& Raichle, 2009); (6) the mean framewise displacement (FD, derived from Jenkinson's relative root-mean-square algorithm) was used to address the residual effects of the residual motion analyses as a covariate between group analyses; and, (7) band-pass filtering between 0.01 and $0.08 \mathrm{~Hz}$.

In global signal regression (GSR), artifactual negative correlations were observed, but this technique was suggested to improve the specificity of positive correlations and to remove specific confounds from the data to facilitate the evaluation of neurophysiological relationships (Fox et al., 2009). For ReHo, most studies have not included GSR in their analyses; for FC, the results with GSR are more readily and reliably interpreted. Based on this information, the GSR step was not included in ReHo but was included in FC analyses in the current study.

The registered fMRI volumes with the Montreal Neurological Institute template were divided into 116 regions according to the automated anatomical labeling Atlas. The Atlas divides the cortex into 90 regions (45 in each hemisphere) and divides the cerebellum into 26 regions (nine in each cerebellar hemisphere and eight in the vermis).

\section{Support vector machine analyses using regional brain features (ReHo)}

The classification algorithm (SVM) was applied using the Pattern Recognition for Neuroimaging Toolbox (PRoNTo) (Schrouff, Rosa, Rondina, \& Marquand, 2013) (http://www. mlnl.cs.ucl.ac.uk/pronto) to estimate potential brain regions contributing most to classifying IGD versus RGU subjects. Briefly, the main steps of the SVM method include: (a) extracting and selecting features, (b) selecting discriminative regions, (c) training the SVM classifier model using the training data, and, (d) evaluating the performance of the SVM model using the evaluation data (Amarreh, Meyerand, Stafstrom, Hermann, \& Birn, 2014; Dyrba, Grothe, Kirste, \& Teipel, 2015).

In the present study, each 3D image was transformed into a column vector of features, and each value corresponded to a single corresponding voxel intensity. Thus, this feature vector encoded the pattern of $\mathrm{ReHo}$ values. In comparison, feature selection involves the selection of a subset of features that facilitates learning (Noble, 2006; Orrù, Pettersson-Yeo, Marquand, Sartori, \& Mechelli, 2012). In this study, feature selection consisted of identifying brain regions that are expected to differ between groups. The above-mentioned procedures were automatically processed in PRoNTos 'Prepare feature set' programs.

A leave-one-out cross-validation method was conducted to perform SVM classifier validation, where feature selection was performed on the training partition of the data each time to avoid circularity effects. In this study, feature selection involved excluding a single subject from each group and training the classifier using the remaining subjects. Then, the excluded subject pair was used to test the ability of the classifier to classify new cases reliably. The abovementioned procedures were repeated for each subject pair until the classifier could obtain a relatively unbiased estimate of generalizability (Orrù et al., 2012). The above procedures were automatically processed in PRoNTos "Specify model" programs. The entire process has been described in detail in previous studies (Schnyer, Clasen, Gonzalez, \& Beevers, 2017).

As for performance evaluation, once the SVM algorithm was established, it was used to predict to which group a new and previously unseen subject would belong (Orrù et al., 2012). A 1,000-times non-parametric permutation test (Cui, Xia, Su, Shu, \& Gong, 2016; Ecker et al., 2010; Schnyer et al., 2017) was used to obtain a corrected p-value to determine the statistical significance of the accuracy, sensitivity and specificity. In detail, accuracy is the proportion of subjects correctly classified into the patient or control group. Sensitivity and specificity represent the proportion of subjects classified correctly. In addition, receiver operating characteristic (ROC) analysis and the AUC (area under the ROC curve) were used to evaluate the performance of the classifiers. The AUC represents the classification power of a 
classifier, and a larger AUC indicates better classification ability (Cui et al., 2016; Fawcett, 2005).

Each model in PRoNTo calculates images representing the weights per voxel and images summarizing the weights per regions of interest, as defined by an atlas (Schrouff et al., 2013). The regional contributions can be ranked in descending order, yielding a sorted list of regions according to their contribution to the classification model. To investigate the classification power of specific locations in the brain, we computed vector weights and listed brain regions with weights in the first $5 \%$, cluster size $>100$ voxels.

\section{SVM analyses using interregional brain features (FC)}

For FC, the SVM was applied using LibSVM, a library for SVMs (https://www.csie.ntu.edu.tw/ cjlin/libsvm/). Based on the LibSVM, the estimation of indexes and the final estimation were performed using scripts (we include these scripts in the Supplementary Materials). The detailed parameters are described below.

We obtained the regional mean time series for each individual by averaging the fMRI time series over all voxels in each of the 116 regions. Pearson correlation coefficient was used in evaluating the FC between each pair of regions. A total of $(116 \times 115) / 2=6,670$ functional connectivity matrix was extracted as classification features for each subject.

Abnormal FC patterns in IGD subjects could be represented by highly discriminating functional connections, thus, by reducing the number of features could accelerates the computation and reduces noise. For this, we first performed 'feature selection' to construct the feature space for classification. Second, we used the Kendall tau rank correlation coefficient to estimate the discriminative power of a feature, which can be quantitatively measured by its relevance to the classification. By these steps, we can calculate a distribution-free test of independence between two variables.

Leave-one-out cross-validation strategy was used to estimate the generalization ability of the classifiers. The final feature set differs slightly between iterations, as the feature ranking is based on a slightly different training data set in each iteration of the cross-validation (the 'one' leaved-out is different for each time). We abstracted the consensus FC of each crossvalidation iteration, and denoted the consensus FC discriminative power by averaging all iterations of the cross-validation. We denoted the region weights (the relative contribution to the identification) by calculating the occurrence number in the consensus functional connections.

(The scripts to run these above-described steps are included as a "feature selection" in the Supplementary files).

After obtaining the features with high discriminative power (25 in the current study, as obtained from the abovementioned step), we employed the SVM with a linear kernel function to solve the classification problem. The best parameter setting was reported as the final results.

Permutation tests were employed to estimate the statistical significance of the observed classification accuracy. In permutation testing, the class labels of the training data were randomly permuted prior to training. Cross-validation was then performed on the permuted training set, and the permutation was repeated 1,000 times.

(The scripts to run "SVM classification" and "permutation test" steps are included as "svm classification" in Supplementary files).

\section{Ethics}

The experiment conforms to the Code of Ethics of the World Medical Association (Declaration of Helsinki). The Human Investigations Committee of Zhejiang Normal University approved this research. All subjects were university students from Shanghai and were recruited through advertisements. All participants provided written informed consent before experimentation.

\section{RESULTS}

\section{ReHo results}

Classifier evaluation results with DSM-5 scores $\geq 5$. In the classification of the two groups, the area under the ROC (AUC) was 0.74 (Fig. 1A). Using a leave-one-out crossvalidation strategy, the linear SVM classifier achieved an accuracy of $73.8 \%$ (43.92\% for IGD; $93.36 \%$ for RGU) when using subjects with scores $\geq 5$ in the DSM- 5 criteria. This result indicates that the SVM identified most IGD subjects as RGU (Fig. 1B and C).

Classifier evaluation results with DSM-5 scores $\geq 6$. In this classification, the area under the ROC (AUC) was 0.95 (Fig. 2A). When using a more stringent threshold for selecting IGD subjects (DSM-5 scores $\geq 6$ ), the classifier achieved an accuracy of $89.84 \%$ (63.44\% for IGD; $98.58 \%$ for RGU). The permutation test showed that the accuracy of this classifier was significantly higher than that achieved by chance ( $P=0.001 ; 1,000$ permutation), which suggests that the SVM can differentiate the two groups to a significant degree (Fig. 2B and C).

Brain regions with high discriminative power. Using the second criteria (DSM-5 score $\geq 6$ ), the most informative regions for classification between IGD subjects and RGUs included the following: (1) bilateral inferior cerebellum (AAL Label 104, 103); OFC (Label 6, 5); cuneus (Label 45); inferior temporal gyrus (Label 89); middle frontal gyrus (Label 8); and parahippocampal (Label 39). See details in Supplementary Table 1.

\section{FC results}

Classification results using DSM-5 scores $\geq 5$. Fig. $3 \mathrm{~A}$ indicates that using 25 indexes achieved the best results. Using leave-one-out cross validation, the linear SVM classifier achieved an accuracy of $60.42 \%$ (41.33\% for IGD; $72.16 \%$ for RGU). The permutation distribution of the estimate is 

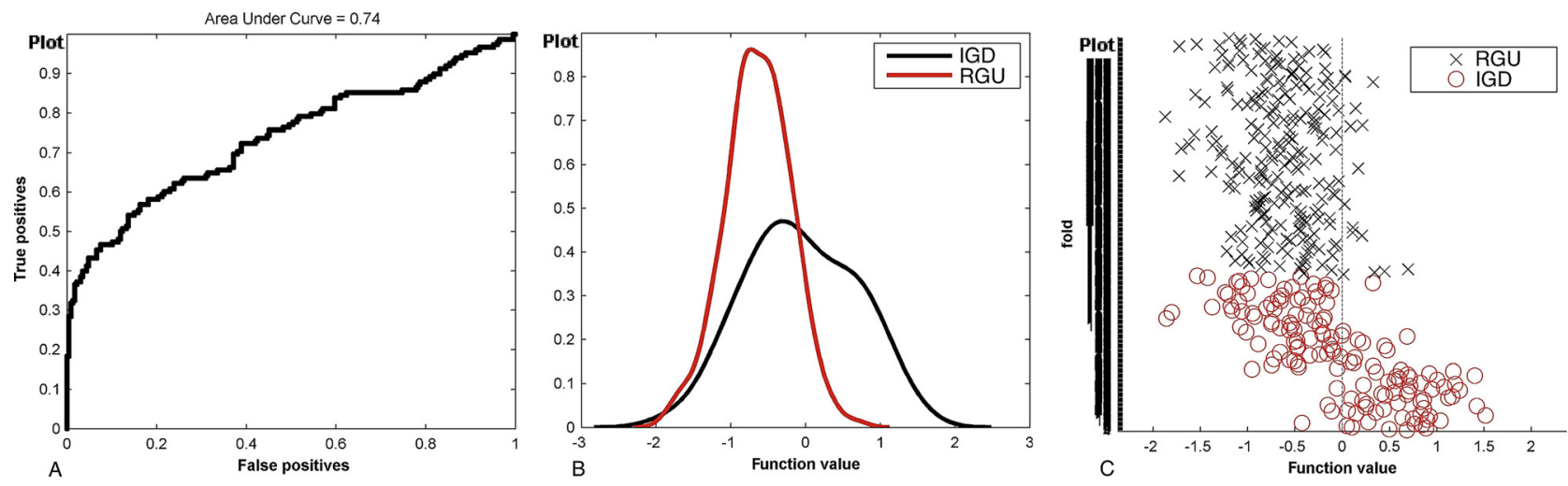

Fig. 1. Classification results using ReHo features with the IGD DSM-5 inclusion criteria $\geq 5$. (A) Area under the curve (AUC; or receiver operating characteristic (ROC)) showing the performance of the binary classifier. (B) Histogram showing the distribution of the two groups.

(C) Scatterplots showing discrimination between the two groups
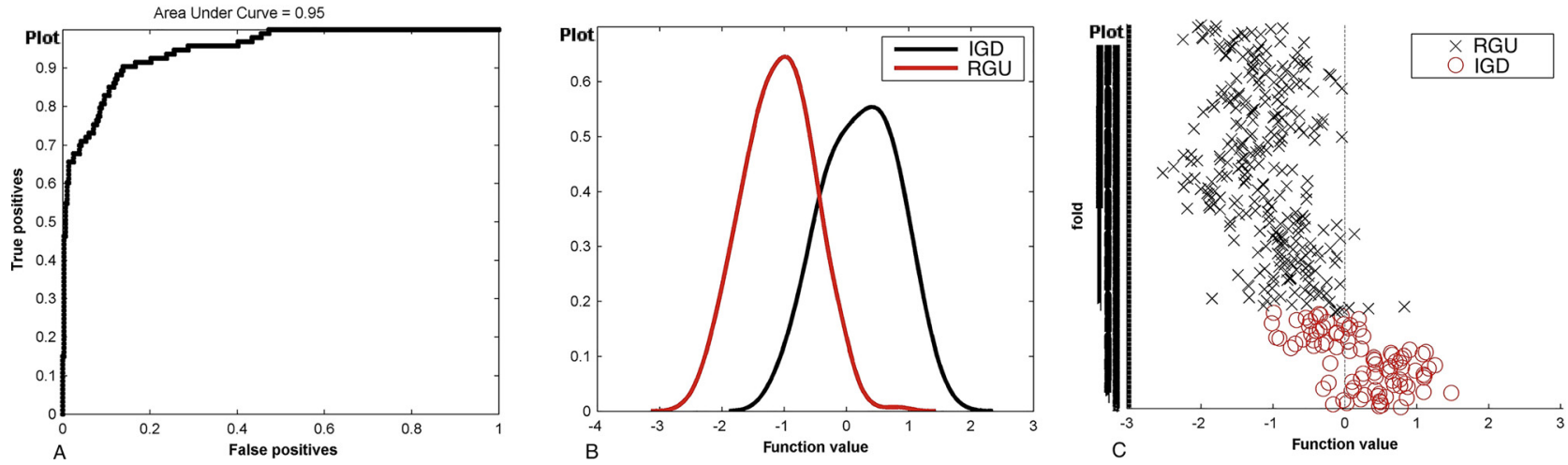

Fig. 2. Classification results using ReHo features with the IGD DSM-5 inclusion criteria $\geq 6$. (A) Area under the curve (AUC; or receiver operating characteristic (ROC)) showing the performance of the binary classifier. (B) Histogram showing the distribution of the two groups.

(C) Scatterplots showing discrimination between the two groups
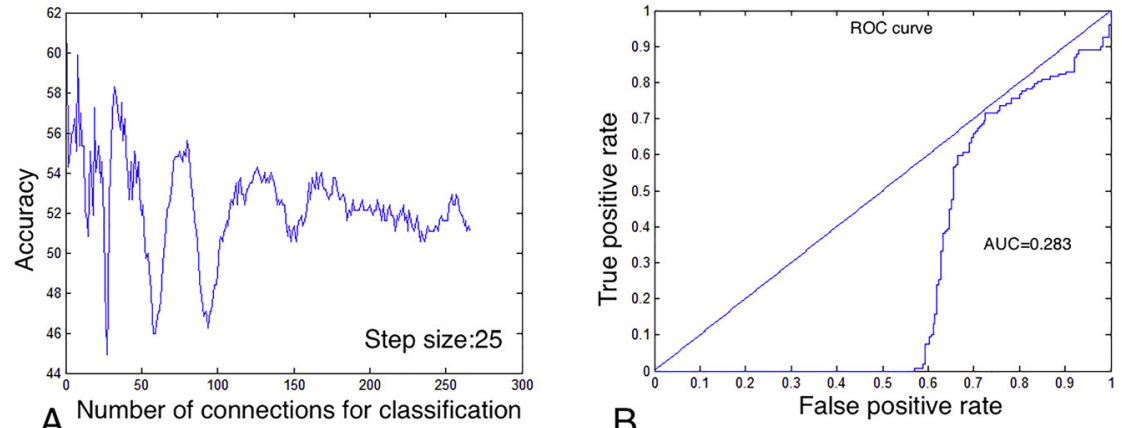

Fig. 3. Classification results using FC features with the IGD DSM-5 inclusion criteria $\geq 5$. (A) Estimated estimation accuracy with a step size $=25$. (B) Area under the curve (AUC; or receiver operating characteristic (ROC)) curve showing the performance of the binary classifier

shown in Fig. 3B, indicating that the classifier did not learn the relationships between the data and labels and was likely to be wrong. The accuracy for this classifier is worse than that expected by random chance, which means that the SVM could not well discriminate the two groups.

Classification results using DSM-5 scores $\geq 6$. Given concerns that IGD may be pathologizing RGU and the poor ability of SVM to distinguish IGD and RGU groups using the proposed diagnostic threshold, we next examined the extent to which increasing the threshold to 6 criteria may improve MVPAs ability to accurately distinguish IGD and RGU groups. Fig. 4A indicates that using 25 indexes achieved the best results. Using leave-one-out cross validation, the linear SVM classifier achieved an accuracy of $75.31 \%$ (59.16\% for IGD; $82.73 \%$ for RGU). The permutation test of 


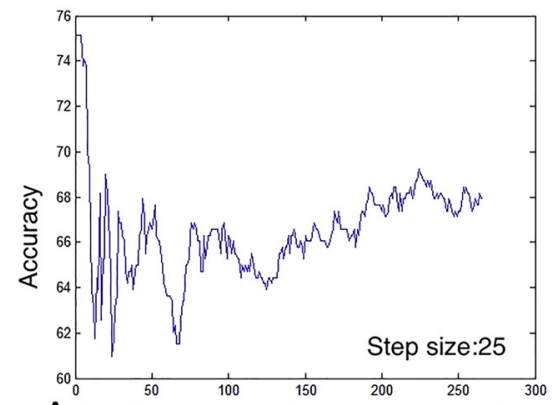

A Number of connections for classification

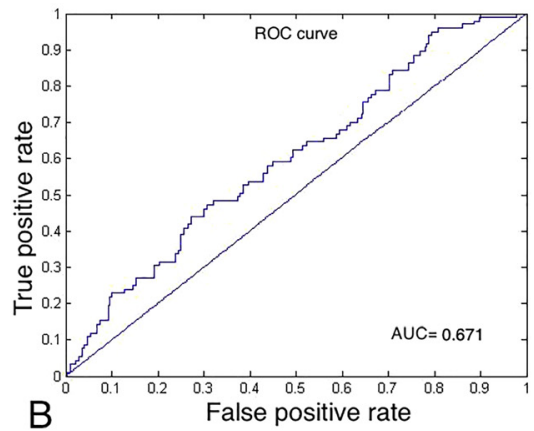

Fig. 4. Classification results using FC features with the IGD DSM-5 inclusion criteria $\geq 6$. (A) Estimated estimation accuracy with a step size $=25$. (B) Area under the curve (AUC; or receiver operating characteristic (ROC)) showing the performance of the binary classifier

the estimate is shown in Fig. 4B, indicating that the classifier learned the relationships between the data and labels and was likely to be correct.

Brain networks with high discriminative power. Supplementary Table 2 shows FC that exhibited higher weights in the classification process. These included regions in the default-mode network (precuneus and middle temporal gyrus) and the executive-control network (ACC and middle frontal cortex).

\section{Reproducibility processing}

To avoid effects from the templates, we also used other templates based on resting states, including the Yeo 17 functional networks template (Yeo et al., 2011). All results showed that SVM could not distinguish IGD from RGU subjects when using a cut-off of $\geq 5$ (accuracy for IGD subjects $=52.03 \%$; when using IGD $\geq 6$, the accuracy rates for IGD subjects increased to $60 \%$, higher than that expected by random chance. See details in Supplementary Materials).

In this study, we used a linear SVM classifier when attempting to discriminate IGD from RGU subjects to determine if MVPA could differentiate the IGD and RGU groups. We found that when using the proposed DSM threshold of $\geq 5$ inclusion criteria, MVPA accuracy rates were lower than those expected by chance, which means that MVPA could not reliably detect differences between IGD and RGU groups. When we used a more stringent threshold (DSM-5 score $\geq 6$ ), MVPA could discriminate the IGD subjects from RGU at a higher rate than that expected by chance, although accuracy was still rather low and could not be used in clinical diagnosis. We believe these conclusions are valid for the following reasons. (1) We investigated regional features and FC as classifying vectors, and both suggested similar conclusions. (2) In addition to structural ROIs (AAL template), we also used brain networks originally from functionally defined regions, which also achieved similar results. (3) Furthermore, choosing the generalization rate as the statistic, the statistical significance of the observed classification accuracies was estimated by permutation testing.

\section{DISCUSSION}

\section{On the diagnostic criteria for IGD}

MVPA cannot reliably distinguish IGD from RGU subjects when using DSM-5 criteria scores $\geq 5$. When using DSM-5 scores $\geq 5$ as inclusion criteria, MVPA could not reliably distinguish IGD from RGU subjects, whether using regional brain features (ReHo) or FC or through use of different templates. In terms of specificity, based on ReHo features, the accuracy rates for IGD subjects (43.92\%) were lower than those expected by random chance (50\%), which suggests that MVPA categorized most of the IGD subjects as RGU during classification. Similar features were also observed when using FC in MVPA. The accuracy rates for IGD subjects $(41.33 \%)$ were also lower than those expected by random chance, and MVPA also categorized most of the IGD subjects as RGU during classification.

According to the theoretical underpinnings of MVPA, when the classifier can label new samples with better-thanchance accuracy, then the two populations are likely to be truly different, and the classifier can capture the populationrelated differences (Golland \& Fischl, 2003). However, in the current situation, MVPA could not distinguish IGD from RGU subjects, which suggests that the two populations demonstrate similar neural features, and MVPA could not capture the differences between these two groups.

To avoid template bias, we also tried the 17 functional networks defined by Yeo et al. (2011). Using these functional regions of interest, SVM also achieved a classification accuracy that was worse-than-random.

Taken together, MVPA could not capture differences between IGD and RGU when using the proposed DSM-5 diagnostic threshold; it more frequently categorized IGD subjects as RGU. These results challenge the diagnostic thresholding for IGD. If, as the current results suggest, there is no biological difference clearly distinguishing IGD and RGU groups, the findings raise questions regarding the current criteria and threshold employed in the DSM-5.

MVPA can distinguish IGD from RGU subjects when using DSM-5 scores $\geq 6$. When we increased the stringency of the IGD inclusion criteria to DSM-5 scores $\geq 6$, the classification 
accuracy improved. Using ReHo, the accuracy rates for IGD subjects reached $63.44 \%$, which is significantly higher than expected by chance $(P=0.001)$, which suggests that MVPA can discriminate IGD from RGU subjects. Using FC in MVPA, the accuracy rates for IGD reached $59.16 \%$, which is also higher than that expected by chance $(P=0.033)$, albeit not as robust as for ReHo. According to the theoretical underpinnings of MVPA, the current results suggest that MVPA can successfully distinguish IGD from RGU subjects at the more stringent IGD threshold, and MVPA can capture differences between these two groups.

We can draw several conclusions from the results. First, MVPA could distinguish IGD from RGU subjects at a more stringent diagnostic threshold, which suggests that IGD subjects show different brain features than RGU. This lends support to the notion that IGD constitutes a specific psychiatric disorder that can be identified using machine learning using neurobiological data.

Second, although MVPA can differentiate IGD from RGU subjects at a more stringent diagnostic threshold, the accuracy rates were approximately $60 \%$, which are higher than those expected by chance but still considerably lower than ideal accuracies for use in clinical settings (using an accuracy rates higher than $85 \%$ may have good predictive power). The results suggest that although the linear SVM classifier learned the relationship between the groups and classifies them with a probability higher than that expected by chance, MVPA may not reliably capture disorder-related resting-state patterns, particularly when using the proposed diagnostic threshold. This result might suggest that resting-state differences are still not that significant between IGD and RGU subjects, particularly when using the proposed diagnostic threshold.

A summary of the results with different inclusion criteria. Taking these two criteria into consideration, we can conclude that, first, IGD subjects show different features than RGU, which could be detected by MVPA when using a threshold of DSM- 5 scores $\geq 6$. This finding supports the notion that IGD can be recognized as a specific disorder because it has specific neural features (both regional features and FC) distinguished from those of RGU, providing support that IGD should be considered an independent disorder rather than a concern secondary to other psychopathology (van Rooij et al., 2018).

However, second, the above conclusions are based on a threshold of DSM-5 scores $\geq 6$. When using the proposed diagnostic criteria $(\geq 5)$, MVPA cannot distinguish the two groups. This suggests that the current diagnostic criteria may not be stringent enough, and more stringent inclusion criteria may be needed. In the current study, although MVPA could distinguish IGD from RGU subjects using resting-state data and a more stringent diagnostic threshold, the accuracy rates are still too low to be used in clinical settings.

\section{Altered resting states in brain regions and networks in IGD}

Cerebellum. Altered connections were observed in the cerebellum and in cerebellar connections with visual and default-mode networks. Studies have reported abnormalities in the cerebellum associated addictive disorders (Miquel et al., 2016; Moreno-Rius \& Miquel, 2017). We speculate that aberrant cerebellar connectivity with the default-mode network and affective network may partially underlie emotional and cognitive symptoms seen in IGD.

Executive-control network. Executive-control networks are widely studied in addictions, including IGD (Brand et al., 2019). IGD patients show impairments in executive-control functions, and in brain regions usually designated as within executive-control networks (Brand et al., 2019; Dong, Wang, Du, \& Potenza, 2018; Zheng et al., 2019). In the current study, the ReHo results showed that the OFC and the middle frontal cortex regions were heavily weighted; the FC results showed FC between the middle frontal cortex/ACC and precuneus, and thalamus. The current results are consistent with other findings implicating executive-control networks in IGD.

Default-mode network. The default-mode network has been suggested to support a baseline state of brain activity. Abnormal function in the default-mode network has been reported in neuropsychiatric disorders including schizophrenia, attention-deficit hyperactivity disorder, depression, Alzheimer's disease and drug addictions (Greicius et al., 2007; Pomarol-Clotet et al., 2008). Abnormalities in the default-mode network in IGD have been reported in several previous studies (L. Wang et al., 2016; Y. Wang et al., 2017; J. T. Zhang et al., 2017).

A summary of altered brain regions and altered FC between brain regions. Both the $\mathrm{ReHo}$ and FC results found that executive-network and default-mode networks were heavily weighted when differentiating IGD from RGU subjects. These results are consistent with previous studies of IGD. However, in previous studies, reward-circuit-related brain regions were often identified in the development and maintenance of IGD; however, the current study did not find a role for these regions in MVPA using resting-state data. This result suggests that IGD involves executive-control failures relating to resting-state function, consistent with prior findings. The extent to which MVPA may implicate reward-related regions/networks using other data (e.g., functional data from cue-reactivity tasks) requires further study.

Limitations and future directions. First, all subjects were from China, and the results need validation using subjects from other countries. Second, we only used resting-state fMRI data. Further studies are needed using task-related brain features or structural features. Third, we did not measure cardiac or respiratory rates in the current study, and these features may have been related to the low-frequency domain of fMRI signals (Murphy et al., 2009) and influenced the conclusions. Fourth, our consideration of IGD criteria treated each similarly, and it is possible that specific criteria may relate to neurobiological features more closely than others. Fifth, criteria for IGD were investigated, 
and the extent to which the findings may extend to criteria for ICD-11 gaming disorder warrant direct investigation. Sixth, multi-modal imaging and clinical data might provide better classification results for IGD and should be investigated in future studies. Seventh, as the DSM-5 diagnostic criteria are still in improving, and the final classification results are based on the DSM-5 proposed criteria. Which might affect the final conclusion of the current study.

\section{CONCLUSIONS}

Based on the findings above, we can draw several conclusions. (1) MVPA can discriminate IGD from RGU subjects based on resting-state fMRI and when using a more stringent diagnostic threshold and can do so with an accuracy higher than that expected by chance; these findings provide support that IGD should be regarded as a legitimate mental disorder, consistent with the World Health Organization's inclusion of gaming disorder in the ICD-11. (2) The DSM-5 diagnostic threshold warrants further consideration, particularly as related to resting-state neural functioning. (3) The majority of the most discriminating brain regions/functional connections were located within or in connection with the cerebellum and executive-control and default-mode networks, thereby indicating that disease-related resting-state network alterations may give rise to some mood and executive-control disturbances seen in IGD.

Funding sources: Dr. Dong was supported by the Zhejiang Natural Science foundation (LY20C090005); Dr. Potenza was supported by the National Center for Responsible Gaming, the Connecticut Council on Problem Gambling, and the Connecticut Department of Mental Health and Addiction Services.

Authors' contribution: Guangheng Dong designed the task and wrote the first draft of the manuscript. Ziliang Wang, Haohao Dong, Yanbin Zheng, Shuer Ye analyzed the data. Min Wang, Jialin Zhang and Xiaoxia Du collected the data. Marc Potenza contributed in editing, interpretation and revision processes. All authors contributed to and have approved the final manuscript.

Conflict of interest: The authors declared that no competing interests exist. Over the past three years, Dr. Potenza has received financial support (to Yale or personally) for the following. He has consulted for and advised Rivermend Health, Game Day Data, Addiction Policy Forum, and Opiant Therapeutics; received research support from the Mohegan Sun Casino and the National Center for Responsible Gaming; consulted for or advised legal and gambling entities on issues related to impulse control and addictive behaviors; provided clinical care related to impulse-control and addictive behaviors; performed grant reviews; edited journals/journal sections; given academic lectures in grand rounds, CME events and other clinical/scientific venues; and generated books or chapters for publishers of mental health texts.

\section{SUPPLEMENTARY MATERIAL}

Supplementary data to this article can be found online at https://doi.org/10.1556/2006.2020.00065.

\section{REFERENCES}

Aarseth, E., Bean, A. M., Boonen, H., Colder Carras, M., Coulson, M., Das, D., et al. (2017). Scholars' open debate paper on the World Health Organization ICD-11 gaming disorder proposal. Journal of Behavioral Addictions, 6(3), 267-270. https://doi.org/ 10.1556/2006.5.2016.088.

Amarreh, I., Meyerand, M. E., Stafstrom, C., Hermann, B. P., \& Birn, R. M. (2014). Individual classification of children with epilepsy using support vector machine with multiple indices of diffusion tensor imaging. Neuroimage Clinical, 4(C), 757-764.

Biswal, B. B., Mennes, M., Zuo, X. N., Gohel, S., Kelly, C., Smith, S. M., et al. (2010). Toward discovery science of human brain function. Proceedings of the National Academy of Sciences of the United States of America, 107(10), 4734-4739. https://doi.org/ 10.1073/pnas.0911855107.

Brand, M., Wegmann, E., Stark, R., Muller, A., Wolfling, K., Robbins, T. W., et al. (2019). The Interaction of Person-AffectCognition-Execution (I-PACE) model for addictive behaviors: Update, generalization to addictive behaviors beyond internetuse disorders, and specification of the process character of addictive behaviors. Neuroscience \& Biobehavioral Reviews, 104, 1-10. https://doi.org/10.1016/j.neubiorev.2019.06.032.

Bruin, W., Denys, D., \& van Wingen, G. (2019). Diagnostic neuroimaging markers of obsessive-compulsive disorder: Initial evidence from structural and functional MRI studies. Progress in Neuro-Psychopharmacology \& Biological Psychiatry, 91, 4959. https://doi.org/10.1016/j.pnpbp.2018.08.005.

Cai, W., Griffiths, K., Korgaonkar, M. S., Williams, L. M., \& Menon, V. (In Press). Inhibition-related modulation of salience and frontoparietal networks predicts cognitive control ability and inattention symptoms in children with ADHD. Molecular Psychiatry. https://doi.org/10.1038/s41380-019-0564-4.

Chen, C. Y., Yen, J. Y., Wang, P. W., Liu, G. C., Yen, C. F., \& Ko, C. H. (2016). Altered functional connectivity of the insula and nucleus accumbens in internet gaming disorder: A resting state fMRI study. European Addiction Research, 22(4), 192-200. https://doi.org/10.1159/000440716.

Cox, L. S., Tiffany, S. T., \& Christen, A. G. (2001). Evaluation of the brief questionnaire of smoking urges (QSU-brief) in laboratory and clinical settings. Nicotine \& Tobacco Research Official Journal of the Society for Research on Nicotine \& Tobacco, 3(1), 7-16.

Craddock, R. C., Holtzheimer, P. E., 3rd, Hu, X. P., \& Mayberg, H. S. (2009). Disease state prediction from resting state functional connectivity. Magnetic Resonance in Medicine, 62(6), 16191628. https://doi.org/10.1002/mrm.22159. 
Cui, Z., Xia, Z., Su, M., Shu, H., \& Gong, G. (2016). Disrupted white matter connectivity underlying developmental dyslexia: A machine learning approach. Human Brain Mapping, 37(4), 14431458.

Cyr, M., Yang, X., Horga, G., \& Marsh, R. (2018). Abnormal fronto-striatal activation as a marker of threshold and subthreshold Bulimia Nervosa. Human Brain Mapping, 39(4), 1796-1804. https://doi.org/10.1002/hbm.23955.

Deleuze, J., Nuyens, F., Rochat, L., Rothen, S., Maurage, P., \& Billieux, J. (2017). Established risk factors for addiction fail to discriminate between healthy gamers and gamers endorsing DSM-5 Internet gaming disorder. Journal of Behavioral Addictions, 6(4), 516-524. https://doi.org/10.1556/2006.6.2017. 074.

Dong, G., Huang, J., \& Du, X. (2011). Enhanced reward sensitivity and decreased loss sensitivity in internet addicts: An fMRI study during a guessing task. Journal of Psychiatric Research, 45(11), 1525-1529. https://doi.org/10.1016/j.jpsychires.2011.06. 017.

Dong, G., Liu, X., Zheng, H., Du, X., \& Potenza, M. N. (2019). Brain response features during forced break could predict subsequent recovery in internet gaming disorder: A longitudinal study. Journal of Psychiatric Research, 113, 17-26. https://doi.org/10. 1016/j.jpsychires.2019.03.003.

Dong, G., \& Potenza, M. N. (2014). A cognitive-behavioral model of Internet gaming disorder: Theoretical underpinnings and clinical implications. Journal of Psychiatric Research, 58, 7-11. https://doi.org/10.1016/j.jpsychires.2014.07.005.

Dong, G., Wang, L., Du, X., \& Potenza, M. N. (2018). Genderrelated differences in neural responses to gaming cues before and after gaming: Implications for gender-specific vulnerabilities to Internet gaming disorder. Social Cognitive and Affective Neuroscience, 13(11), 1203-1214. https://doi.org/10.1093/scan/ nsy084.

Dong, G., Wang, M., Liu, X., Liang, Q., Du, X., \& Potenza, M. N. (In Press). Cue-elicited craving-related lentiform activation during gaming deprivation is associated with the emergence of Internet gaming disorder. Addiction Biology. https://doi.org/10. 1111/adb.12713.

Dong, G., Wang, Z., Wang, Y., Du, X., \& Potenza, M. N. (2019) Gender-related functional connectivity and craving during gaming and immediate abstinence during a mandatory break: Implications for development and progression of internet gaming disorder. Progress In Neuro-Psychopharmacology \& Biological Psychiatry, 88, 1-10. https://doi.org/10.1016/j.pnpbp.2018.04.009.

Dong, G. H., Wang, M., Wang, Z., Zheng, H., Du, X., \& Potenza, M. N. (2020). Addiction severity modulates the precuneus involvement in internet gaming disorder: Functionality, morphology and effective connectivity. Progress in Neuro-Psychopharmacology \& Biological Psychiatry, 98, 109829. https:// doi.org/10.1016/j.pnpbp.2019.109829.

Dong, G. H., Wang, M., Zhang, J., Du, X., \& Potenza, M. N. (2019). Functional neural changes and altered cortical-subcortical connectivity associated with recovery from Internet gaming disorder. Journal of Behavioral Addictions, 8(4), 692-702. https://doi.org/10.1556/2006.8.2019.75.

Dong, G. H., Wang, M., Zheng, H., Wang, Z., Du, X., \& Potenza, M. N. (2020). Disrupted prefrontal regulation of striatum-related craving in Internet gaming disorder revealed by dynamic causal modeling: Results from a cue-reactivity task. Psychological Medicine, 1-13. https://doi.org/10.1017/S003329172000032X.

Dong, G., Zheng, H., Liu, X., Wang, Y., Du, X., \& Potenza, M. N. (2018). Gender-related differences in cue-elicited cravings in Internet gaming disorder: The effects of deprivation. Journal of Behavioral Addictions, 7(4), 953-964. https://doi.org/10.1556/ 2006.7.2018.118.

Dyrba, M., Grothe, M., Kirste, T., \& Teipel, S. J. (2015). Multimodal analysis of functional and structural disconnection in Alzheimer's disease using multiple kernel SVM. Human Brain Mapping, 36(6), 2118-2131.

Ecker, C., Rocha-Rego, V., Johnston, P., Mourao-Miranda, J., Marquand, A., Daly, E. M., et al. (2010). Investigating the predictive value of whole-brain structural MR scans in autism: A pattern classification approach. NeuroImage, 49(1), 44-56.

Erickson, B. J., Korfiatis, P., Akkus, Z., \& Kline, T. L. (2017). Machine learning for medical imaging. RadioGraphics, 37(2), 505515. https://doi.org/10.1148/rg.2017160130.

Fawcett, T. (2005). An introduction to ROC analysis. Pattern Recognition Letters, 27(8), 861-874.

Fox, M. D., Zhang, D., Snyder, A. Z., \& Raichle, M. E. (2009). The global signal and observed anticorrelated resting state brain networks. Journal of Neurophysiology, 101(6), 3270-3283. https://doi.org/10.1152/jn.90777.2008.

Golland, P., \& Fischl, B. (2003). Permutation tests for classification: Towards statistical significance in image-based studies. Information Processing in Medical Imaging, 18, 330-341. Retrieved from http://www.ncbi.nlm.nih.gov/pubmed/15344469.

Greicius, M. D., Flores, B. H., Menon, V., Glover, G. H., Solvason, H. B., Kenna, H., et al. (2007). Resting-state functional connectivity in major depression: Abnormally increased contributions from subgenual cingulate cortex and thalamus. Biological Psychiatry, 62(5), 429-437. https://doi.org/10.1016/j.biopsych. 2006.09.020.

Griffiths, M. D., van Rooij, A. J., Kardefelt-Winther, D., Starcevic, V., Kiraly, O., Pallesen, S., et al. (2016). Working towards an international consensus on criteria for assessing internet gaming disorder: A critical commentary on Petry et al. (2014). Addiction, 111(1), 167-175. https://doi.org/10.1111/add.13057.

Han, X., Wang, Y., Jiang, W., Bao, X., Sun, Y., Ding, W., et al. (2018). Resting-state activity of prefrontal-striatal circuits in internet gaming disorder: Changes with cognitive behavior therapy and predictors of treatment response. Frontiers in Psychiatry, 9, 341. https://doi.org/10.3389/fpsyt.2018.00341.

Ioannidis, K., Hook, R., Goudriaan, A. E., Vlies, S., Fineberg, N. A., Grant, J. E., et al. (2019). Cognitive deficits in problematic internet use: meta-analysis of 40 studies. British Journal of Psychiatry, 215(5), 1-8. https://doi.org/10.1192/bjp.2019.3.

Kardefelt-Winther, D., Heeren, A., Schimmenti, A., van Rooij, A., Maurage, P., Carras, M., et al. (2017). How can we conceptualize behavioural addiction without pathologizing common behaviours?. Addiction, 112(10), 1709-1715. https://doi.org/10. 1111/add.13763.

Kim, J. Y., Chun, J. W., Park, C. H., Cho, H., Choi, J., Yang, S., et al. (2019). The correlation between the frontostriatal network and impulsivity in internet gaming disorder. Scientific Reports, 9(1), 1191. https://doi.org/10.1038/s41598-018-37702-4. 
Kiraly, O., \& Demetrovics, Z. (2017). Inclusion of Gaming Disorder in ICD has more advantages than disadvantages. Journal of Behavioral Addictions, 6(3), 280-284. https://doi.org/10.1556/ 2006.6.2017.046.

Lecrubier, Y., Sheehan, D. V., Weiller, E., Amorim, P., Bonora, I., Harnett Sheehan, K., et al. (1997). The Mini international neuropsychiatric interview (MINI). A short diagnostic structured interview: Reliability and validity according to the CIDI. European Psychiatry, 12(5), 224-231. Retrieved from http:// www.sciencedirect.com/science/article/B6VM1-3W7PVMS-PS/ 2/934fceb2414d46c20210beef880acd59.

Mihara, S., \& Higuchi, S. (2017). Cross-sectional and longitudinal epidemiological studies of internet gaming disorder: A systematic review of the literature. Psychiatry and Clinical Neurosciences, 71(7), 425-444. https://doi.org/10.1111/pcn. 12532.

Miquel, M., Vazquez-Sanroman, D., Carbo-Gas, M., Gil-Miravet, I., Sanchis-Segura, C., Carulli, D., et al. (2016). Have we been ignoring the elephant in the room? Seven arguments for considering the cerebellum as part of addiction circuitry. Neuroscience \& Biobehavioral Reviews, 60, 1-11. https://doi. org/10.1016/j.neubiorev.2015.11.005.

Moreno-Rius, J., \& Miquel, M. (2017). The cerebellum in drug craving. Drug and Alcohol Dependence, 173, 151-158. https:// doi.org/10.1016/j.drugalcdep.2016.12.028.

Murphy, C. A., Blyth, K. G., Chaudhuri, R., Lafferty, J., Hothersall, E., Steedman, T., et al. (2009). Assessment of the presence of occult myocardial infarction in chronic obstructive pulmonary disease using contrast-enhanced cardiac magnetic resonance imaging. Respiration, 78(3), 263-269. https://doi.org/10.1159/000203354.

Noble, W.S. (2006). What is a support vector machine? Nature Biotechnology, 24(12), 1565-1567.

Orrù, G., Pettersson-Yeo, W., Marquand, A. F., Sartori, G., \& Mechelli, A. (2012). Using support vector machine to identify imaging biomarkers of neurological and psychiatric disease: A critical review. Neuroscience \& Biobehavioral Reviews, 36(4), $1140-1152$.

Palaus, M., Marron, E. M., Viejo-Sobera, R., \& Redolar-Ripoll, D. (2017). Neural basis of video gaming: A systematic review. Frontiers in Human Neuroscience, 11. https://doi.org/10.3389/ fnhum.2017.00248.

Park, S. M., Lee, J. Y., Choi, A. R., Kim, B. M., Chung, S. J., Park, M., et al. (In Press). Maladaptive neurovisceral interactions in patients with Internet gaming disorder: A study of heart rate variability and functional neural connectivity using the graph theory approach. Addiction Biology, e12805. https://doi.org/10. 1111/adb.12805.

Petry, N. M., \& O’Brien, C. P. (2013). Internet gaming disorder and the DSM-5. Addiction, 108(7), 1186-1187. https://doi.org/10. 1111/add.12162.

Petry, N. M., Rehbein, F., Gentile, D. A., Lemmens, J. S., Rumpf, H. J., Mossle, T., et al. (2014). An international consensus for assessing internet gaming disorder using the new DSM-5 approach. Addiction, 109(9), 1399-1406. https://doi.org/10. 1111/add.12457.

Petry, N. M., Rehbein, F., Gentile, D. A., Lemmens, J. S., Rumpf, H. J., Mossle, T., et al. (2016). Griffiths et al.'s comments on the international consensus statement of internet gaming disorder:
Furthering consensus or hindering progress? Addiction, 111(1), 175-178. https://doi.org/10.1111/add.13189.

Pomarol-Clotet, E., Salvador, R., Sarro, S., Gomar, J., Vila, F., Martinez, A., et al. (2008). Failure to deactivate in the prefrontal cortex in schizophrenia: Dysfunction of the default mode network? Psychological Medicine, 38(8), 1185-1193. https://doi. org/10.1017/S0033291708003565.

Potenza, M. N. (2018). Do gaming disorder and hazardous gaming belong in the ICD-11? Considerations regarding the death of a hospitalized patient that was reported to have occurred while a care provider was gaming. Journal of Behavioral Addictions, 7(2), 206-207. https://doi.org/10.1556/2006.7.2018.42.

Przybylski, A. K., Weinstein, N., \& Murayama, K. (2017). Internet gaming disorder: Investigating the clinical relevance of a new phenomenon. American Journal of Psychiatry, 174(3), 230-236. https://doi.org/10.1176/appi.ajp.2016.16020224.

Quandt, T. (2017). Stepping back to advance: Why IGD needs an intensified debate instead of a consensus. Journal of Behavioral Addictions, 6(2), 121-123. https://doi.org/10.1556/2006.6.2017.014.

Rehbein, F., Kliem, S., Baier, D., Mossle, T., \& Petry, N. M. (2015). Prevalence of Internet gaming disorder in German adolescents: Diagnostic contribution of the nine DSM- 5 criteria in a statewide representative sample. Addiction, 110(5), 842-851. https:// doi.org/10.1111/add.12849.

Schnyer, D. M., Clasen, P. C., Gonzalez, C., \& Beevers, C. G. (2017). Evaluating the diagnostic utility of applying a machine learning algorithm to diffusion tensor MRI measures in individuals with major depressive disorder. Psychiatry Research, 264, 1-9.

Schrouff, J., Rosa, M. J., Rondina, J. M., \& Marquand, A. F. (2013). PRoNTo: Pattern recognition for neuroimaging toolbox. Neuroinformatics, 11(3), 319-337.

Strittmatter, E., Parzer, P., Brunner, R., Fischer, G., Durkee, T., Carli, V., et al. (2016). A 2-year longitudinal study of prospective predictors of pathological Internet use in adolescents. European Child \& Adolescent Psychiatry, 25(7), 725-734. https://doi.org/10.1007/s00787-015-0779-0.

Tian, L., Ren, J., \& Zang, Y. (2012). Regional homogeneity of resting state fMRI signals predicts Stop signal task performance. NeuroImage, 60(1), 539-544. https://doi.org/10.1016/j.neuroimage.2011.11.098.

van den Eijnden, R., Koning, I., Doornwaard, S., van Gurp, F., \& Ter Bogt, T. (2018). The impact of heavy and disordered use of games and social media on adolescents' psychological, social, and school functioning. Journal of Behavioral Addictions, 7(3), 697-706. https://doi.org/10.1556/2006.7.2018.65.

van den Heuvel, M. P., \& Hulshoff Pol, H. E. (2010). Exploring the brain network: A review on resting-state fMRI functional connectivity. European Neuropsychopharmacology, 20(8), 519534. https://doi.org/10.1016/j.euroneuro.2010.03.008.

Van Rooij, A. J., \& Kardefelt-Winther, D. (2017). Lost in the chaos: Flawed literature should not generate new disorders. Journal of Behavioral Addictions, 6(2), 128-132. https://doi.org/10.1556/ 2006.6.2017.015.

van Rooij, A. J., Ferguson, C. J., Colder Carras, M., Kardefelt-Winther, D., Shi, J., Aarseth, E., et al. (2018). A weak scientific basis for gaming disorder: Let us err on the side of caution. Journal of Behavioral Addictions, 7(1), 1-9. https://doi.org/10.1556/2006.7.2018.19.

Wang, Z., Dong, H., Du, X., Zhang, J. T., \& Dong, G. H. (2020). Decreased effective connection from the parahippocampal 
gyrus to the prefrontal cortex in Internet gaming disorder: A MVPA and spDCM study. Journal of Behavioral Addictions, 9(1), 105-115. https://doi.org/10.1556/2006.2020.00012.

Wang, M., Dong, H., Zheng, H., Du, X., \& Dong, G. H. (2020). Inhibitory neuromodulation of the putamen to the prefrontal cortex in Internet gaming disorder: How addiction impairs executive control. Journal of Behavioral Addictions, 9(2), 312324. https://doi.org/10.1556/2006.2020.00029.

Wang, R., Li, M., Zhao, M., Yu, D., Hu, Y., Wiers, C. E., et al. (2019). Internet gaming disorder: Deficits in functional and structural connectivity in the ventral tegmental area-accumbens pathway. Brain Imaging and Behavior, 13(4), 1172-1181. https://doi.org/10.1007/s11682-018-9929-6.

Wang, Z., Liu, X., Hu, Y., Zheng, H., Du, X., \& Dong, G. (2019). Altered brain functional networks in Internet gaming disorder: Independent component and graph theoretical analysis under a probability discounting task. CNS Spectrums, 24(5), 544-556. https://doi.org/10.1017/S1092852918001505.

Wang, L., Wu, L., Lin, X., Zhang, Y., Zhou, H., Du, X., et al. (2016). Dysfunctional default mode network and executive control network in people with Internet gaming disorder: Independent component analysis under a probability discounting task. European Psychiatry, 34, 36-42. https://doi.org/10.1016/j.eurpsy. 2016.01.2424.

Wang, Y., Wu, L., Luo, L., Zhang, Y., \& Dong, G. (2017). Shortterm Internet search using makes people rely on search engines when facing unknown issues. PLoS One, 12(4), e0176325. https://doi.org/10.1371/journal.pone.0176325.

Wang, Z., Wu, L., Yuan, K., Hu, Y., Zheng, H., Du, X., et al. (2018). Cortical thickness and volume abnormalities in Internet gaming disorder: Evidence from comparison of recreational Internet game users. European Journal of Neuroscience, 48(1), 16541666. https://doi.org/10.1111/ejn.13987.

Wang, M., Zeng, N., Zheng, H., Du, X., Potenza, M. N., \& Dong, G. H. (In Press). Altered effective connectivity from the pregenual anterior cingulate cortex to the laterobasal amygdala mediates the relationship between internet gaming disorder and loneliness. Psychological Medicine, 1-10. https://doi.org/10.1017/S0033291720002366.

Wartberg, L., Kriston, L., Zieglmeier, M., Lincoln, T., \& Kammerl, R. (2019). A longitudinal study on psychosocial causes and consequences of Internet gaming disorder in adolescence. Psychological Medicine, 49(2), 287-294. https://doi.org/10.1017/ S003329171800082X.

Woo, C.-W., Chang, L. J., Lindquist, M. A., \& Wager, T. D. (2017). Building better biomarkers: Brain models in translational neuroimaging. Nature Neuroscience, 20(3), 365-377.
Yan, C. G., Chen, X., Li, L., Castellanos, F. X., Bai, T. J., Bo, Q. J., et al. (2019). Reduced default mode network functional connectivity in patients with recurrent major depressive disorder. Proceedings of the National Academy of Sciences of the United States of America, 116(18), 9078-9083. https://doi.org/10.1073/pnas.1900390116.

Yan, C. G., Wang, X. D., Zuo, X. N., \& Zang, Y. F. (2016). DPABI: Data processing \& analysis for (Resting-State) brain imaging. Neuroinformatics, 14(3), 339-351. https://doi.org/10.1007/ s12021-016-9299-4.

Yao, Y. W., Liu, L., Ma, S. S., Shi, X. H., Zhou, N., Zhang, J. T., et al. (2017). Functional and structural neural alterations in Internet gaming disorder: A systematic review and meta-analysis. Neuroscience \& Biobehavioral Reviews, 83, 313-324. https://doi. org/10.1016/j.neubiorev.2017.10.029.

Yeo, B. T., Krienen, F. M., Sepulcre, J., Sabuncu, M. R., Lashkari, D., Hollinshead, M., et al. (2011). The organization of the human cerebral cortex estimated by intrinsic functional connectivity. Journal of Neurophysiology, 106(3), 1125-1165. https://doi.org/ 10.1152/jn.00338.2011.

Young, K. S. (2009, Jan. 2010). Internet addiction test (IAT). Retrieved from http://netaddiction.com/index.php?option= combfquiz\&view $=$ onepage\&catid $=46 \&$ Itemid $=106$.

Yuan, K., Yu, D., Cai, C., Feng, D., Li, Y., Bi, Y., et al. (2017). Frontostriatal circuits, resting state functional connectivity and cognitive control in internet gaming disorder. Addiction Biology, 22(3), 813-822. https://doi.org/10.1111/adb.12348.

Zang, Y., Jiang, T., Lu, Y., He, Y., \& Tian, L. (2004). Regional homogeneity approach to fMRI data analysis. NeuroImage, 22(1), 394-400. https://doi.org/10.1016/j.neuroimage.2003.12.030.

Zeng, L. L., Shen, H., Liu, L., Wang, L., Li, B., Fang, P., et al. (2012). Identifying major depression using whole-brain functional connectivity: A multivariate pattern analysis. Brain, 135(Pt 5), 1498-1507. https://doi.org/10.1093/brain/aws059.

Zhang, J. T., Ma, S. S., Yan, C. G., Zhang, S., Liu, L., Wang, L. J., et al. (2017). Altered coupling of default-mode, executive-control and salience networks in Internet gaming disorder. European Psychiatry, 45, 114-120. https://doi.org/10.1016/j.eurpsy.2017.06.012.

Zhang, Y., Tian, J., Yuan, K., Liu, P., Zhuo, L., Qin, W., et al. (2011). Distinct resting-state brain activities in heroin-dependent individuals. Brain Research, 1402(2011), 46-53.

Zheng, H., Hu, Y., Wang, Z., Wang, M., Du, X., \& Dong, G. (2019). Meta-analyses of the functional neural alterations in subjects with Internet gaming disorder: Similarities and differences across different paradigms. Progress in Neuro-Psychopharmacology \& Biological Psychiatry, 94, 109656. https://doi.org/10. 1016/j.pnpbp.2019.109656. 